Classification

Physics Abstracts

72.20F $-72.20 \mathrm{M}-73.60 \mathrm{~F}$

\title{
Annealing-related electrical and piezoresistive properties of B- and As-implanted LPCVD silicon films
}

\author{
P. Jeanjean ( $\left.{ }^{1}\right)$, J. Sicart $\left({ }^{1}\right)$, J. L. Robert $\left({ }^{1}\right)$, M. Le Berre $\left({ }^{2}\right)$, P. Pinard $\left({ }^{2}\right)$ and \\ V. Conedera $\left({ }^{3}\right)$ \\ (1) Groupe d'Etude des Semiconducteurs, UA 357, USTL, 34095 Montpellier Cedex 05, France \\ $\left({ }^{2}\right)$ Laboratoire de Physique de la Matière, LA 358, INSA, 69621 Villeurbanne, France \\ (3) Laboratoire Automatisme et Analyse des Systèmes, CNRS, 31077 Toulouse, France
}

(Received 4 June 1992, accepted 17 September 1992)

\begin{abstract}
Résumé. - Nous étudions les propriétés électriques de films silicium polycristallin implantés au bore ou à l'arsenic. Deux types de recuit post-implantation ont été effectués : un recuit conventionnel (CTA) et un recuit rapide (RTA). Nous montrons que le recuit RTA améliore les propriétés électriques. Les facteurs de gauge ont été déterminés et nous concluons que le recuit RTA est adapté à la technologie des capteurs intégrés silicium.

Abstract. - We investigate the electrical properties of polycrystalline silicon thin films implanted with arsenic or boron ions. Two types of post-implantation annealing have been performed on the samples : a conventional thermal annealing (CTA) and a rapid thermal annealing (RTA). We find that RTA improves the electrical properties. Measurements of gauge factors have been carried out on both films. We conclude that RTA can be applied successfully in thin film silicon integrated sensor technology.
\end{abstract}

\section{Introduction.}

Polycrystalline (pc) silicon is commonly used for many years in sensor applications because its compatibility with silicon integrated circuits makes possible integrated realization of sensors with electronics. The Low Pressure Chemical Vapor Deposition (LPCVD) provides thin films of pc-silicon deposited on oxidized silicon wafers which serves as active layers or thin film substrates in SOI (Silicon On Insulator) technology. Depending on the application, the dopant concentration varies between $10^{17} \mathrm{~cm}^{-3}$ (thin films transistors) and $10^{20} \mathrm{~cm}^{-3}$ (mechanical sensors). The implantation technique is applied for doping in microelectronics and an annealing step is required to activate the dopant species and remove the implantation damage. In this paper, we focus on the influence of the post-implantation annealing on both electrical and piezoresistive properties of LPCVD polycrystalline films implanted with arsenic or boron ions. 
Two types of annealing were performed on the wafers : a Conventional Thermal Annealing (CTA) using a standard furnace anneal and a Rapid Thermal Annealing (RTA) using an incoherent beam source. A set of galvanomagnetic measurements were performed to compare the dopant activation, temperature coefficient of resistance (TCR) and Hall mobility in various samples. Piezoresistivity measurements were achieved to compare the gauge factors for pressure sensor applications.

Several authors [1] have previously investigated the electrical and piezoresistive properties of implanted LPCVD films. The post-implantation annealing was a standard CTA $\left(950{ }^{\circ} \mathrm{C}\right.$ $\cdot 1000^{\circ} \mathrm{C}, 20-30 \mathrm{mn}$ ) and we will compare our results with theirs. We complete the measurements with data collected after RTA. We conclude on the efficiency of this novel annealing technique in SOI technology for mechanical sensors.

\section{Electrical properties.}

2.1 Materials AND METHODS. - The pc-silicon films were deposited on thermally oxidized CZ Si substrates by LPCVD. The LOCOS (Local Oxide) and pc-silicon thicknesses were $710 \mathrm{~nm}$ and $420 \mathrm{~nm}$ respectively. A low temperature oxide cap, $400 \mathrm{~nm}$ thick, prevented dopant exodiffusion during further annealing. The layers were boron $(30 \mathrm{keV})$ or arsenic $\left(100 \mathrm{keV}\right.$ ) implanted with the doses (I) $5 \times 10^{15}$, (II) $8 \times 10^{14}$, and (III) $4 \times 10^{14} \mathrm{~cm}^{-2}$, respectively. A rapid thermal annealing (RTA, $1100^{\circ} \mathrm{C}, 20 \mathrm{~s}$ ) was performed in Ar ambient using an automatic monitored processor (Sitesa-Addax $\mathrm{R} 1000$ ). The heating and cooling rates were $125^{\circ} \mathrm{C} / \mathrm{s}$ and $80^{\circ} \mathrm{C} / \mathrm{s}$ respectively. To compare RTA films with CTA films, a standard furnace anneal (CTA, $950^{\circ} \mathrm{C}, 30 \mathrm{mn}$ ) in $\mathrm{N}_{2}$ ambient was performed on the implanted LPCVD layers. The samples were patterned in Hall bars $\left(100 \times 1000 \mu \mathrm{m}^{2}\right)$ with 6-terminals. A further annealing $\left(450^{\circ} \mathrm{C}, 20 \mathrm{mn}\right)$ in $\mathrm{N}_{2}+7 \% \mathrm{H}_{2}$ ambient was achieved on all the samples after $\mathrm{Al}$ contact metallization.

Electrical measurements were performed using a current supply (Keithley 220) and by reversing both the current $I$ and the magnetic field $B(1 \mathrm{~T})$ during Hall measurements. The sheet resistance $R_{\mathrm{sq}}$ and the carrier concentration $n_{\mathrm{H}}$ were given by :

$$
\begin{aligned}
& R_{\mathrm{sq}}=\frac{\pi f}{I \ln 2} \frac{\sum_{1}^{n} V_{\mathrm{t}}}{n} \\
& n_{\mathrm{H}}=\frac{A}{q} \frac{I B}{V_{\mathrm{H}} t}
\end{aligned}
$$

where $V_{H}$ is the Hall voltage, $n$ the number of Van der Pauw configurations and $t$ the film thickness. Since the samples behaved as quasi-degenerated semiconductors and showed no asymetric effect, the correction coefficient $f$ was taken as equal to unity. The factor $A$ is the anisotropy factor for silicon. It was taken to be 0.87 in As-implanted films and 0.75 in B-implanted films respectively [2]. The galvanomagnetic measurements were achieved between $4 \mathrm{~K}$ and $400 \mathrm{~K}$ using a He flow regulated cryostat Oxford CF200.

2.2 EXPERIMENTAL RESUlTS. - Tables I and II give experimental data (dopant electrical efficiency, mobility) measured at room temperature. They are similar to those published elsewhere [3-5]. Series I behaved as a degenerated semiconductor film with low TCR (i.e. with a carrier concentration and mobility quasi-constant w ith temperature). Series II and III showed a thermally activated mobility characteristic of a polycrystalline semiconductor.

The activation energy decreased with temperature as commonly observed in this doping concentration range. As a matter of example figure 1 shows the electrical behaviour exhibited 
Table I. - Experimental results in As-implanted films. $R_{\mathrm{sq}}$ denotes the sheet resistance. \% is the ratio of $n_{\mathrm{H}}$ to the implanted species concentration. TCR denotes the temperature coefficient of resistance calculated between $300 \mathrm{~K}$ and $220 \mathrm{~K} . E_{\mathrm{B}}$ and $N_{\mathrm{t}}$ denote the GB barrier height and trap density respectively. $G_{1}$ and $G_{\mathrm{t}}$ are the longitudinal and transverse gauge factors respectively.

\begin{tabular}{|c|c|c|c|c|c|c|c|c|}
\hline \multicolumn{1}{|c|}{ Sample } & $R_{\mathrm{sq}}$ & As activation & Mobility & TCR & $E_{\mathrm{b}}$ & $N_{\mathrm{t}}$ & $G_{1}$ & $G_{\mathrm{t}}$ \\
\hline \multicolumn{1}{|c|}{ Units } & Ohm & $\%$ & $\mathrm{~cm}^{2} / \mathrm{Vs}$ & $\mathrm{ppm} /{ }^{\circ} \mathrm{C}$ & $\mathrm{meV}$ & $10 \mathrm{E} 12 \mathrm{~cm}^{-2}$ & - & - \\
\hline CTA I & 262 & 22.2 & 24.6 & -1925 & 20 & 5.2 & -19.5 & 4.7 \\
\hline CTA II & 3690 & 18.3 & 13.5 & -8011 & 36 & 2.5 & -20.5 & 1.1 \\
\hline CTA III & 22857 & 13.3 & 6 & -19149 & 56 & 1.9 & -20.5 & -2.1 \\
\hline RTA I & 104 & 49.8 & 28 & -882 & 15.5 & 6.8 & -19.8 & 4 \\
\hline RTA II & 1471 & 35.2 & 15.1 & -7068 & 39 & 3.6 & - & - \\
\hline RTA III & 5571 & 28.6 & 10.2 & -12522 & 47.5 & 2.55 & -17 & 1.4 \\
\hline
\end{tabular}

Table II. - Experimental results in B-implanted films. Labels are the same as in table I.

\begin{tabular}{|c|c|c|c|c|c|c|c|c|}
\hline Sample & $R_{\mathrm{sq}}$ & B activation & Mobılity & \multicolumn{1}{c|}{ TCR } & $E_{\mathrm{b}}$ & $N_{\mathrm{t}}$ & $G_{1}$ & $G_{\mathrm{t}}$ \\
\hline \multicolumn{1}{|c|}{ Units } & Ohm & $\%$ & $\mathrm{~cm}^{2} / \mathrm{Vs}$ & $\mathrm{ppm} /{ }^{\circ} \mathrm{C}$ & $\mathrm{meV}$ & $10 \mathrm{E} 12 \mathrm{~cm}^{-2}$ & - & - \\
\hline CTA I & 117 & 54.4 & 25.7 & 23 & 10 & 5.7 & 30 & -5 \\
\hline CTA II & 1145 & 60 & 15.3 & -2319 & 20 & 3.4 & 33 & -6 \\
\hline CTA III & 4214 & 43.4 & 11.5 & -5353 & 31 & 2.5 & - & - \\
\hline RTA I & 56 & 84 & 35.7 & 487 & 7 & 6.4 & 28 & -4.7 \\
\hline RTA II & 450 & 74.6 & 31 & -1212 & 17 & 3.5 & 43 & -5.3 \\
\hline RTA III & 1707 & 67 & 18.3 & -3456 & 24 & 2.8 & 40 & -2.8 \\
\hline
\end{tabular}

by films implanted with $A$ s or $B$ at the same dose (I) respectively. It can be seen that :

i) RTA implementes both the dopant activation and the mobility;

ii) RTA films show a lower TCR than CTA films;

iii) B-implanted films have performances higher than As-implanted films.

To understand such a behaviour, we interpret the experimental results in the framework of the trapping model of Seto [6]. In this model, defects at grain boundaries (GB) act as trapping states $N_{\mathrm{t}}$ for the majority carriers. Then a potential barrier $E_{\mathrm{b}}$ built up at GBs leads to an additionnal scattering mechanism for the majority carriers in the conduction band. Consequently, the Hall mobility is strongly reduced and controlled by conduction at GBs. Otherwise, the grain boundaries are depleted from majority carriers such that almost all carriers which contribute to the Hall voltage are located in the grain. Thus they have to overcome the GB barrier to contribute to the conductivity. Then GBs act as symetric double Schottky barriers and Seto applied the well known thermoemission formalism leading to the following 


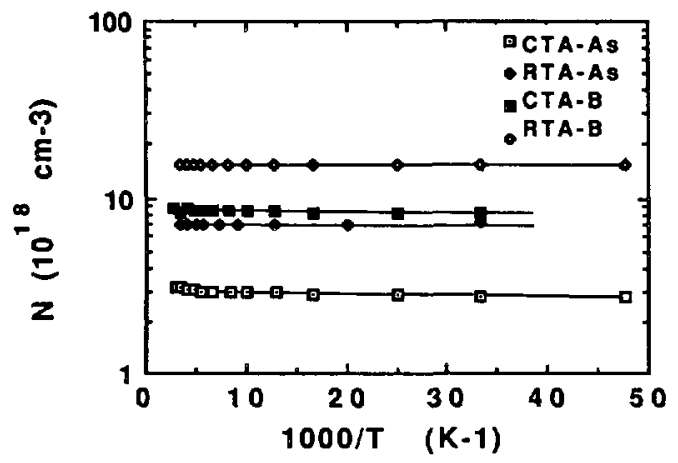

a)

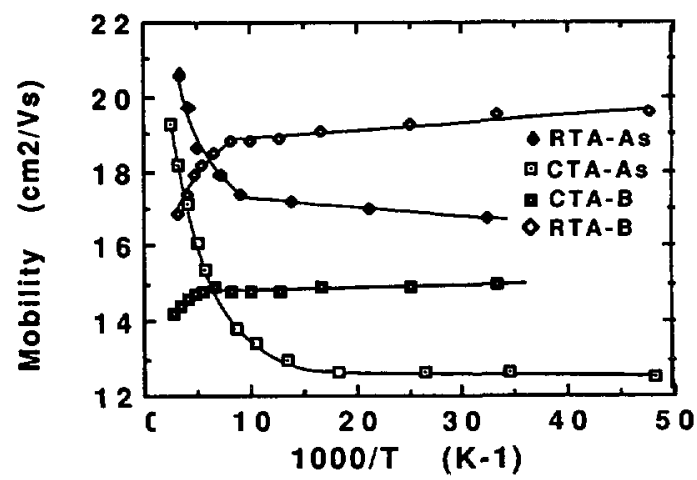

b)

Fig. 1. - Hall data $(A=1)$ in samples implanted with the dose $5 \times 10^{15} \mathrm{~cm}^{-2}$ (series I). a) carrier concentration. b) mobility vs. $1000 / T$.

expressions :

$$
\begin{aligned}
E_{\mathrm{B}} & =\frac{q^{2}}{8 \varepsilon} \frac{N_{\mathrm{t}}^{2}}{N_{\mathrm{d}}} \\
\mu & =q L(2 \pi m k T)^{-1 / 2} \exp \left(-E_{\mathrm{B}} / k T\right)
\end{aligned}
$$

where $N_{\mathrm{d}}$ is the dopant concentration and $L$ the grain size. Then, the potential barrier at GBs is responsible for the low and thermally activated mobility in polycrystalline silicon.

We calculated the barrier height $E_{\mathrm{B}}$ and the density $N_{\mathrm{t}}$ of trapping centers at GBs using equation (4) and equation (3) respectively. In equation (3) we replaced the dopant concentration $N_{\mathrm{d}}$ by the actual carrier concentration $N$ given by equation (2) taking into account the factor $A$ (n-type and p-type semiconductor respectively) since the dopant activation was not complete. Table I gives values for $N_{\mathrm{t}}$ which are similar for the two species and are increasing with the implantation dose.

However, a decrease of $N_{\mathrm{t}}$ in As-implanted CTA films is observed and has been interpreted as segregation of As atoms and complexe formation during the anneal [5, 7]. This segregation effect is negligible in B-implanted films [7]. Equation (3) shows clearly that it is the high electrical activation of dopants in RTA which yields a low GB barrier height $E_{\mathrm{B}}$. This leads of course to a lower TCR in RTA films and an increase of the Hall mobility at low temperatures (Fig. 1). Mobilities of As- and B-implanted films are similar since the value of the preexponential term (Eq. (4)) is a function of the grain size $L$ which is about the same for the two species. Concerning the influence of the annealing on the grain size, we find that RTA improves the mobility. Krause et al. [12] studied the grain growth in RTA and concluded that it was very rapid at the onset of the annealing and then approached the growth kinetics of CTA. In our opinion, the influence of the annealing on the grain size could be studied properly only if the two annealings (RTA and CTA) are performed at the same temperature, which was not the case in our work. However, it is worth mentioning that TEM micrographs performed on LPCVD films implanted with boron and annealed with parameters like ours gave grain sizes in the range $50-200 \mathrm{~nm}$ in RTA films and in the range $50-100 \mathrm{~nm}$ in CTA films [13]. In conclusion, we found that RTA improved the electrical properties of LPCVD films : low resistivity, low TCR, high dopant electrical activation, high mobility. Let us now investigate the piezoresistive properties in order to know if RTA implementes also the gauge factor. 


\section{Piezoresistive properties.}

3.1 MATERIALS AND METHODS. - To compare the resistivity of the silicon films under pressure and to calculate the longitudinal $G_{1}$ and transverse $G_{1}$ gauge factors, Hall bars were patterned along and perpendicular to a silicon beam $\left(3 \times 40 \mathrm{~mm}^{2}\right)$ as shown in figure $2 \mathrm{a}$. Twelve Al wires $50 \mu \mathrm{m}$ wide, patterned along the beam assured electrical connection with the external measurement apparatus. They were ultrasonic bonded to copper plots on a board sticked to the beam and electrically connected with the external set-up. Then the beam was squeezed between two sapphire slices and the beam bending was achieved applying a vertical stress as shown in figure $2 \mathrm{~b}$. The longitudinal/transverse resistances were measured using the 4 in-line probes resistor method and a Keithley 220 current source. The gauge factor was derived from :

$$
\Delta R=G R \Delta \varepsilon
$$

where the microstrain $\Delta \varepsilon$ is calculated from the incremental micrometric displacement $\Delta d$ using the classical bend beam expression :

$$
\Delta \varepsilon=\frac{3}{2} \frac{Z b}{L_{3}} \Delta d
$$

where $L, Z, b$ denote lengths and thickness as shown in figure $2 \mathrm{~b}$.

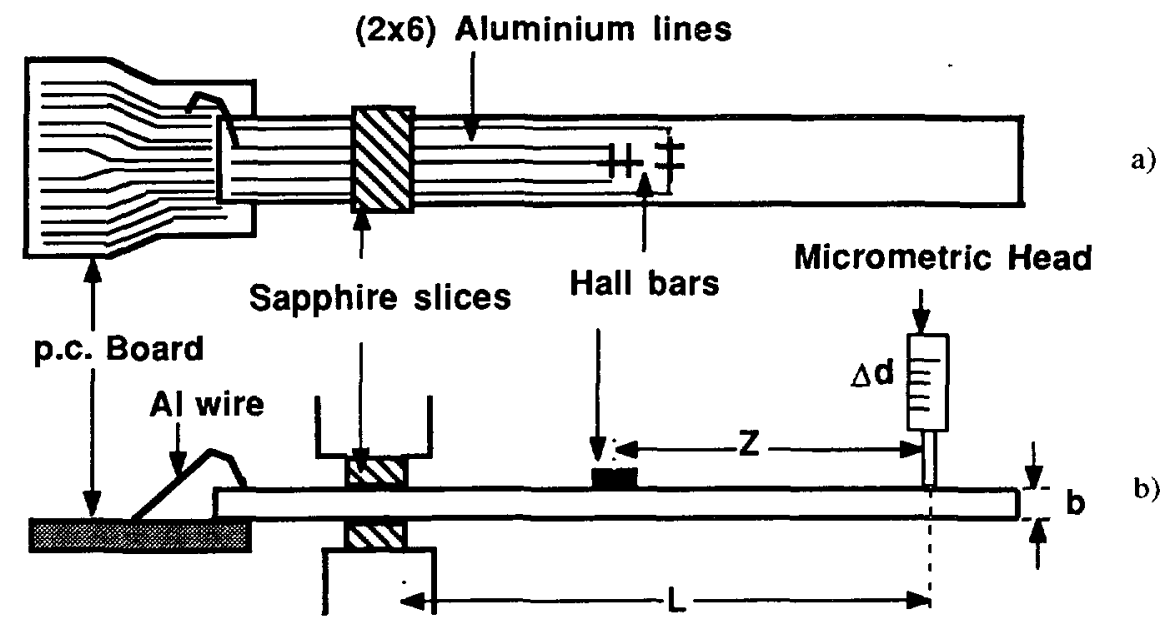

Fig. 2. - Experimental set-up for determining the gauge factors.

3.2 EXPERIMENTAL RESULTS. - Figures 3 and 4 show typical curves for the relative change $\Delta R / R$ versus compressive/tensile microstrain in As-implanted and B-implanted films respectively. Values of the gauge factors $G_{1}$ and $G_{\mathrm{t}}$ at room temperature are collected in table I. The values of the $G$ factor in CTA films are similar to those published previously by other authors $[1,8,9]$. They are lower than $G$ factors in crystalline silicon due to GB effect. Laserrecrystallized silicon films have also higher $G$ factors [10], but only a few data are available in RTA films [11]. Our results show that the $G$ factor in RTA films is close to that in CTA films implanted with the same dose. 


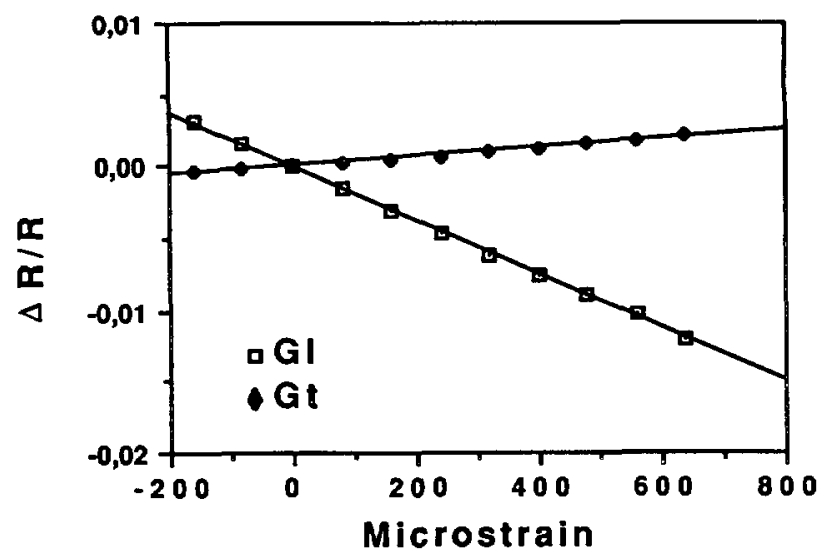

Fig. 3. - Relative change of the resistance vs. the applied microstrain in As-implanted films (series I).

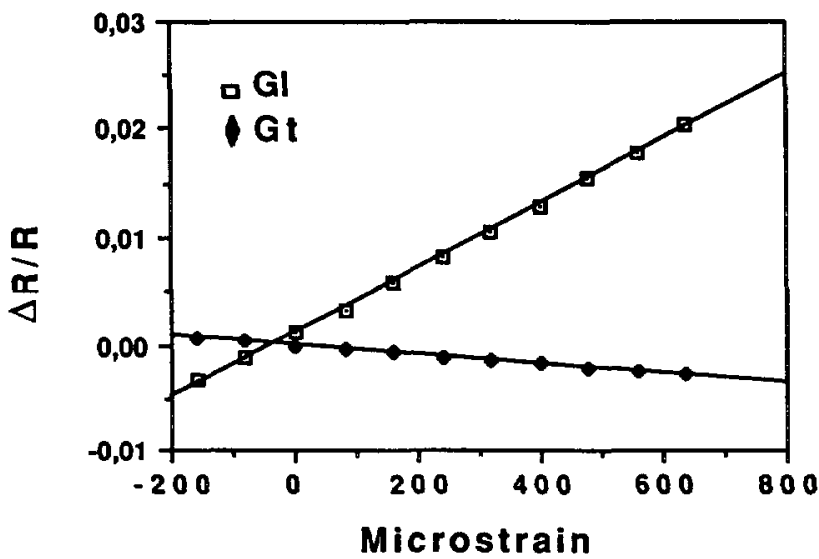

Fig. 4. - Relative change of the resistance vs. the applied microstrain in B-implanted films (series I).

\section{Conclusions.}

LPCVD polysilicon films implanted with arsenic or boron and annealed by CTA or RTA have been investigated. The related galvanomagnetic measurements have shown that RTA improves the electrical properties of the films. The piezoresistive properties for RTA polysilicon pressure or mechanical sensing application have been compared to those of CTA films. We find that the gauge factors are similar for films having the same implantation dose. We conclude that RTA could be an alternative to standard CTA-processed polysilicon pressure sensors. Indeed the fact that RTA implementes the electrical properties would be benefic in an integrated rapid thermal process for SOI monolithic sensors manufacturing. 


\section{Acknowledgements.}

The LPCVD wafers were provided by the CNET (Meylan) and processed by the TEAMLAAS (Toulouse). Mrs. I. Salesse is thanked for the ultrasonic bonding of the samples. The authors would like to thank V. Mosser from the SMR (Montrouge) research group for fruitful discussions. This work was supported by the French GdR on Micronic Integrated Sensors.

\section{References}

[1] Mosser V., Suski J., Goss J. and Obermeier E., Sensors Actuators A28 (1991) 113 and references therein.

[2] BeER A. C., Galvanomagnetic Effects in Semiconductors, Solid State Physics, Vol, 4 (Academic Press, N.Y.) (1963).

[3] Wilson S. R., Gregory R. B., Paulson W. M., Krause S. J., Gresset J. D., Hamdi A. H., Mc Daniel F. D. and Downing R. G., J. Electrochem. Soc. 132 (1985) 922.

[4] Alvi N. S., Tang S. M., Kwor R. and Fulcher M. R., J. Appl. Phys. 62 (1987) 4878.

[5] Jeanjean P., Sellito P., Sicart J., Robert J. L., Chaussemy G. and Laugier A., Semicond. Sci. Technol. 6 (1991) 1130.

[6] Seto J. Y., J. Appl. Phys. 46 (1975) 5247.

[7] Mandurah M. M., Saraswat K. C., Helms C. R. and Kamins T. I., J. Appl. Phys. 51 (1980) 5755.

[8] Schubert D., Jenschke W., Uhlig T. and Schmidt F. M., Sensors Actuators 11 (1987) 145.

[9] French P. J. and Evans A. G. R., Solid State Electron. 32 (1989) 1.

[10] Binder J., Henning W., Obermeier E., Schaber H. and Cutter D., Sensors Actuators 4 (1983) 527.

[11] De la Celle R., Cervera-Marzal M., Geslot P., Mosser V., Suski J. and Goss J., Proc. Capteurs'89, Paris (June 6-9 1989) 96.

[12] Krause S. J., Wilson S. R., Paulson W. M. and Gregory R. B., Appl. Phys. Lett. 45 (1984) 778.

[13] Almaggoussi A., Sicart J., Robert J. L., Chaussemy G. and Laugier A., J. Appl. Phys. 66 (1989) 4301. 